\title{
Association between schizophrenia and periodontal disease in relation to cortisol levels: an ELISA-based descriptive analysis
}

Bariah Fahad Albahli ${ }^{1}$, Najd Mohammed Alrasheed ${ }^{2}$, Raghad Saleh Alabdulrazaq ${ }^{1}$, Dhafer S. Alasmari ${ }^{3}$ and Muzammil Moin Ahmed ${ }^{4 *}$ (1)

\begin{abstract}
Background: Schizophrenia is a chronic psychosis marked by multiple bioenvironmental and immunological dysregulation with its intricate role in etiopathogenesis of periodontal disease remaining unclear. Hence, the aim of this study is to determine the association between periodontal disease and schizophrenia in relation with cortisol levels.

Methods: The study is in descriptive design comprised of 40 subjects randomly selected (20 schizophrenic patients as Group A and 20 healthy volunteers as group B). All the study participants underwent complete periodontal examination including scoring of gingival index (GI), plaque index (PI), Probing depths (PD) and clinical attachment loss (CAL). Salivary cortisol levels are estimated using ELISA. Link between schizophrenia and periodontal disease is described in relation to cortisol levels with elimination of other shared risk factors, such as tobacco smoking and xerostomia.

Results: Significant higher values of periodontal parameters are observed in Group A with schizophrenic patients (GI 2.467 $\pm 0.528 ;$ PI $2.402 \pm 0.526 ;$ PD 2.854 \pm 0.865 ; CAL 1.726 \pm 3.096$)$ than Group B with healthy subjects (Gl $0.355 \pm 0.561 ;$ PI $0.475 \pm 0.678$; PD 1.493 \pm 0.744 ; CAL 0.108 \pm 0.254$)$. However, cortisol levels are lower in schizophrenic group $(0.190 \pm 0.059)$ than non-schizophrenic group $(0.590 \pm 0.228)$ ruling out the possible role of cortisol in periodontal disease severity associated with schizophrenic patients.

Conclusion: Findings of this study, provides ground evidence for consideration of schizophrenia as a risk factor for periodontitis and demands greater emphasis on management of schizophrenic patients in dental setting similar to other comorbid disorders such as diabetes mellitus and also incorporating periodontal care measures in the clinical guidelines for schizophrenia management.
\end{abstract}

Keywords: Schizophrenia, Periodontitis, Cortisol, Mental disorders, Periodontal risk factors, Periodontal medicine, Periodontal pathogenesis

\section{Introduction}

Schizophrenia is a chronic neuropsychotic illness that involves complex interaction of environmental and biogenetic factors. It is characterized by delusions,

\footnotetext{
*Correspondence: muzzu_ahmed@yahoo.co.in; mu.ahmed@qu.edu.sa ${ }^{4}$ Department of Dental Hygiene, College of Applied Health Sciences in Ar Rass, Qassim University, 51921 Al Qassim, Kingdom of Saudi Arabia Full list of author information is available at the end of the article
}

hallucinations, and disintegration of thought processes leading to disorganized speech and emotional responsiveness that occurs in clear consciousness resulting in social and occupational disability and failure to understand reality [1,2]. It influences more than 23 million individuals around the world but isn't as common as numerous other mental disorders and males are more frequently affected than females [3]. The common symptoms of schizophrenia can be negative that include social 
withdrawal, self-neglect, loss of motivation, blunted affect, and paucity of speech and positive symptoms can be hallucination, delusions and lack of insight [2]. People with schizophrenia often have additional mental health problems, such as anxiety, depression or substance-use disorders [4]. Though, typical age of onset is early $20 \mathrm{~s}$, it can affect even children at the age of 5 or 6 [5]. The exact etiology of this neuropsychological disorder is unknown. However, complex interaction of genetic, biological, psychological and social factors might cause this schizophrenic psychosis with psychosocial factors contributing the most $[6,7]$. Mentally ill people suffer, not only from their psychiatric conditions but also from physical impairment and psychosocial domains of their health. [8]

Schizophrenia confronts challenges in understanding and maintaining good oral health. Typical challenges with dental care of patients with mental impairment include difficulties in performing routine home oral hygiene, lack of cooperation during routine dental treatment, inability to comprehend the need for treatment, need for sedation or general anesthesia, oral effects of medication, aggressive behavior and prejudice related oral health care. Periodontal disease and dental caries are frequently seen dental conditions among psychiatric patients. This is largely because oral health is ranked low on the priority list in the context of mental illness.

Periodontal diseases comprise of a group of inflammatory conditions of the supporting tissues of the teeth caused by micro-organisms and their responses by the host. It has been defined as an oppugnancy between the host and periodontal pathogens leading to disruption of host homeostasis and periodontal tissue destruction [9]. Periodontal attachment loss and periodontal pockets are common clinical manifestations of plaque induced progressive periodontal destruction and attachment loss [10]. The presence of periodontal inflammation and destruction are measured and characterized by periodontal clinical parameters, prominent of which are gingival bleeding, alteration in gingival morphological characteristics, consistency of periodontal destruction with amount of dental plaque and measurement of clinical attachment loss and probing depths. Plaque accumulation has a direct effect on clinical attachment loss [11]. Silness and Loe plaque index (PI) is an important parameter to measure the level and rate of plaque formation on tooth surfaces [12]. Gingival inflammation is the prerequisite for the development of periodontitis which can be measured and graded using Loe and Silness gingival index (GI). Gingival index not only detect the gingival inflammation but also clinically characterises its severity [12]. Periodontitis is detected as clinical attachment loss (CAL) which is a prominent determinant of periodontal severity and reflects the destruction of periodontal tissues [13]. Patients related factors such as age and sex have no discernible effect on clinical attachment loss which makes its more dependable in staging the periodontitis [14]. Probing depth (PD) determines the complexity of periodontitis and its measurement is crucial for periodontal disease diagnostic classification [13]. Also, measurement of probing depths using periodontal probe is the only reliable and convenient method of determining the presence of periodontal pockets. [14].

Antipsychotic drugs utilized in schizophrenia can induce xerostomia, which is related with a few dental clutters and the result of hyposalivation is the worsening of periodontal diseases and rapid development of caries $[1,7,15]$. Dysregulation of hypothalamic pituitary adrenal axis (HPA) in schizophrenic patients leads to hypercortisolemia. Cortisol levels are affected by physical stress, emotional stress, and illnesses. Periodontal literature establishes the link between periodontal disease and stress that might result due to some negative life events and psychological factors increasing the susceptibility to periodontal disease [16]. There is an increased prevalence of poor periodontal health among the psychiatric inpatients which could be due to diminished inspiration for oral care, adverse oral effect of antipsychotics such as xerostomia, dysregulation of HPA axis, decreased regular dental visits and dental specialists are not trained to deal with this category of patients [6, 7]. However, periodontal health has been rarely studied with respect to schizophrenia, among the parts of oral health. Thus, the aim of this study was to determine the association between periodontal disease and schizophrenia in relation with salivary cortisol levels.

\section{Methods}

The study was conducted as per the revised 2013 Helsinki declaration for experimentations involving human subjects and approved by ethical research committees of College of Dentistry, Qassim University and Al Amal \& Psychiatric Hospital, Buraidah with number F-3003-18. Subjects aged between 20 and 60 years satisfying the eligibility criteria were included in the study using simple random sampling technique. The study consisted of 40 subjects divided into two groups: Group-A with 20 schizophrenic patents and Group-B with 20 non-schizophrenic healthy patients. Of all the eligible schizophrenic patients, 20 patients were selected by lottery method for periodontal examination and saliva sample collection, whereas 20 healthy subjects were selected from out patients of Qassim University dental clinics. The nature and rationale of the study was explained to all the study participants or to their guardian, and written consent was obtained for the same. Study inclusion criteria were 1. Cooperative Schizophrenic and stressed patients for 
intra-oral examination. 2. Subjects who had at least 20 teeth. The exclusion criteria included 1. Patients who receive radio therapy or taking chemotherapy. 2. Patients suffering from chronic oral diseases, such as malignant tumours and patients on cancer chemotherapy. 3. Pregnant and lactating females. 4. Patients who have received treatment with anti-inflammatory drugs, antibiotics, steroids, hormonal replacement therapy in the recent past. 5. Patients who have undergoing periodontal therapy in past 6 months. 6 . Subjects with drug abuse and tobacco consumption. All the subjects underwent periodontal examination for Loe and Silness gingival index (GI), Silness and Loe plaque index (PI), probing depth (PD) and clinical attachment loss (CAL). UNC-15 probe was used to calculate PD and CAL. Salivary cortisol levels were estimated using enzyme linked immunosorbent assay (ELISA). The saliva samples were collected from the patients using Salivatte (which contains a plain cotton swab) that provides an optimal method for hygienic collection of saliva. Then the saliva was separated from cotton using Hettich Zentrifugen Centrifuge with EBA20 model and type number 2002. ELISA Kit together with Zenyth 3100 multilabel platform (ELISA reader) was used to quantitatively determine the cortisol level in saliva using 96 wells coated with anti-cortisol antibody (polyclonal).

\section{Statistical analysis}

In this study, sample size was calculated using $G^{*}$ power. Difference between two independent means (two groups), was applied with an effect size of 0.8 and an alpha error of 0.05 with $90 \% \mathrm{CI}$. The total sample size is 56, group A ( 28 sample) and group B ( 28 sample). The assessed parameters were statistically analysed using IBM SPSS Statistics version 22.0.0.0. Descriptive statistics such as mean and standard deviation were determined for each group. Independent sample $t$ test was used to compare the difference in mean Cortisol level, the plaque index and the gingival index in between Group A and B. Serum cortisol levels and periodontal health obtained from the gingival index and periodontal index were correlated using Pearson's correlation coefficient ( $\mathrm{R}$ value). If $P<0.05$, then it was considered statistically significant.

\section{Results}

Of 56 calculated sample sizes, samples were collected from 40 subjects with dropout rate of $28.57 \%(n=16)$. The drop out resulted from exclusion of 16 subjects with 8 in each group due to non-cooperation or dry mouth in schizophrenic group and unequal distribution of cases in healthy group. The sociodemographic characteristics of study participants of both the groups are shown in Table 1. There was no significant difference with
Table 1 Comparison of sociodemographic characteristics of study participants

\begin{tabular}{llll}
\hline Variables & Group A & Group B & ' $\boldsymbol{p}^{\text {' value }}$ \\
\hline $\begin{array}{l}\text { Mean Age (Years) } \\
\text { Gender }\end{array}$ & $39.1 \pm 12.1$ & $38.3 \pm 11.6$ & 0.632 \\
$\quad$ Male & $85 \%(n=17)$ & $90 \%(n=18)$ & 0.516 \\
$\quad$ Female & $15 \%(n=3)$ & $10 \%(n=2)$ & \\
Total & $100 \%(n=20)$ & $100 \%(n=20)$ & \\
Marital Status & & & \\
$\quad$ Single & $45 \%(n=9)$ & $60 \%(n=12)$ & 0.141 \\
$\quad$ Married & $55 \%(n=11)$ & $40 \%(n=8)$ & \\
Total & $100 \%(n=20)$ & $100 \%(n=20)$ & \\
Employment & & & \\
$\quad$ Employed & $30 \%(n=6)$ & $95 \%(n=19)$ & 0.000 \\
$\quad$ Unemployed & $70 \%(n=14)$ & $5 \%(n=1)$ & \\
$\quad$ Total & $100 \%(n=20)$ & $100 \%(n=20)$ & \\
\hline
\end{tabular}

regards to age and gender due to the matched selection of healthy subjects. However, there is significant difference in the employment status with higher employment rate in healthy group than the schizophrenic patients which could be attributed to the limitation posed by the mental illness. The mean age of schizophrenia onset was $26.4 \pm 9.6$ years with mean duration of illness as $10.2 \pm 6.7$ years. Group-A schizophrenic patients were on neuroleptics drugs as a long-term therapy and antidepressants were prescribed intermittently. Mean duration of antipsychotic use was $8.98 \pm 4.3$ years.

The statistical analysis comparing periodontal parameters revealed significant higher values of PI, BI, PD and CAL in group-A: schizophrenic patients than group-B: healthy subjects (Table 2). This indicates the increased gingival inflammation and periodontal destruction in schizophrenic patients than the healthy individuals. However, independent sample $t$ test showed lower salivary cortisol levels in group A than group B (Table 2). The intragroup comparison that assessed the correlation between salivary cortisol levels and clinical periodontal parameters, did not show statistically significant correlation between them (Table 3). This finding rules out the possible influence of cortisol in the periodontal tissue inflammation.

\section{Discussion}

Schizophrenia is a common functional psychotic disorder and a chronic psychotic disorder that affects the patient's thoughts causing delusions and hallucinations. In this cross-sectional descriptive study, an attempt was made to explore the possible association between schizophrenia and periodontal diseases and its correlation with cortisol levels. Cortisol is a glucocorticoid steroidal hormone and 
Table 2 Comparison of clinical periodontal parameters and salivary cortisol levels between two groups

\begin{tabular}{|c|c|c|c|c|c|c|c|c|c|c|c|c|}
\hline \multirow[t]{2}{*}{ Variables } & \multicolumn{4}{|c|}{ Group-A } & \multicolumn{4}{|c|}{ Group-B } & \multirow[t]{2}{*}{ Mean difference } & \multirow{2}{*}{$\begin{array}{l}\text { SD error } \\
\text { difference }\end{array}$} & \multirow[t]{2}{*}{ ' $t$ ' value } & \multirow[t]{2}{*}{ ' $p$ 'value } \\
\hline & Min & Max & Mean & SD & Min & Max & Mean & SD & & & & \\
\hline $\mathrm{Gl}$ & 0.50 & 3.00 & 2.467 & 0.528 & 0.000 & 2.000 & 0.355 & 0.561 & 2.11 & 0.172 & 12.236 & 0.000 \\
\hline $\mathrm{PI}$ & 0.50 & 3.00 & 2.402 & 0.526 & 0.000 & 2.000 & 0.475 & 0.678 & 1.927 & 0.192 & 10.039 & 0.000 \\
\hline $\mathrm{PD}(\mathrm{mm})$ & 1.250 & 4.870 & 2.854 & 0.865 & 1.00 & 4.200 & 1.493 & 0.744 & 1.3610 & 0.2552 & 5.330 & 0.000 \\
\hline $\mathrm{CAL}(\mathrm{mm})$ & 0.000 & 14.500 & 1.726 & 3.096 & 0.000 & 1.000 & 0.108 & 0.254 & 1.6189 & 0.6947 & 2.330 & 0.025 \\
\hline Cortisol levels & 0.000 & 0.257 & 0.190 & 0.059 & 0.168 & 1.009 & 0.590 & 0.228 & -0.400 & 0.052 & -7.595 & 0.000 \\
\hline
\end{tabular}

Table 3 Pearson correlation between clinical periodontal parameters and cortisol levels

\begin{tabular}{lll}
\hline Periodontal parameters & \multicolumn{2}{l}{ Salivary cortisol levels } \\
\cline { 2 - 3 } & $\boldsymbol{R}$ value & $\boldsymbol{P}$ value \\
\hline $\mathrm{Gl}$ & 0.208 & 0.378 \\
$\mathrm{PI}$ & 0.214 & 0.364 \\
$\mathrm{PD}$ & 0.027 & 0.910 \\
$\mathrm{CAL}$ & 0.056 & 0.814 \\
\hline
\end{tabular}

secretion of which is controlled by hypothalamic pituitary adrenal axis (HPA). Analysis of cortisol in saliva is highly promising, accurate and non-invasive method for assessment of chronic stress and HPA axis $[17,18]$. A clinical prospective study of Zoja et al., that compared the serum cortisol levels between schizophrenia patients and healthy controls has concluded that elevated serum cortisol levels may be considered as a biomarker for the diagnosis of schizophrenia and may be used as a significant predictor for positive response to antipsychotic treatment in schizophrenia patients with acute exacerbation [19]. According to the results of another study by Kornetova et al., patients who underwent treatment, and does not present notable clinical signs of schizophrenia may have moderately lowered levels of salivary cortisol which is a reflection of relenting psychotic symptoms as well as a direct effect of atypical antipsychotic drugs on HPA axis activity. [6, 20, 21].

In the present study, cortisol levels were lower in schizophrenic patients than healthy patients and there was no correlation between cortisol and clinical periodontal parameters. This could be due to the possible effect of schizophrenic patients undergoing medical treatment which can be explained by the earlier studies on schizophrenic patients that have demonstrated the reduction in cortisol levels of schizophrenic patients following antipsychotic treatment $[22,23]$. A by Rady et al., has found reduction in serum cortisol level of chronic schizophrenic patients on antipsychotics [24]. It is evident from the literature that cortisol has negative effects on periodontal disease progress and severity. Higher cortisol levels are also associated with severity and progression of periodontal disease. However, lower cortisol levels found in our study rules out the possible role of cortisol in periodontal disease severity associated with schizophrenic patients undergoing antipsychotic therapy.

Another finding of this study is increase in indicators of periodontal disease activity such plaque index, gingival index, probing depth and clinical attachment loss in schizophrenic patients than healthy. A study by Shreya et al., has demonstrated increased evidence of poor periodontal condition with higher GI, PI and PD in schizophrenic patients suggesting the possible link between periodontal disease and schizophrenia [6]. However, Shreya et al., have not considered the possible role of cortisol, xerostomia and other risk factors associated with schizophrenia and periodontitis.

Both schizophrenia and periodontal diseases share some of the common risk factors such as stress, xerostomia and tobacco consumption with immunoinflammatory alterations playing major role. Xerostomia has been recognized as an important risk factor for dental diseases and its impact on the quality of life of sufferers [25]. Emotional and psychological alterations and psychotropics induces xerostomia in patients with schizophrenia which may facilitate periodontal inflammation. In schizophrenia patients with chronic use of antipsychotic medications, such as aripiprazole, pernazine and risperidone, have a negative effect on salivary secretions, since salivary glands are neurobiologically regulated by autonomic nervous system, which influence the levels of transmitter substances and thereby affect the salivary gland function that lead to xerostomia which may negatively affect periodontal health [26, 27]. Eltas et al., reported that the side effects of antipsychotic medications and smoking caused severe periodontal disease in psychiatric patients [28]. However, confounding factors such as xerostomia and tobacco consumption have been eliminated by subject selection criteria to rule out their possible influence on periodontal status focusing the role of cortisol.

The relationship between periodontal disease and schizophrenia could be bilateral. Host homeostatic 
changes induced by schizophrenia may enhance the severity and progression of periodontal disease. Periodontal disease may in turn influence the pathological course of schizophrenia through contributing to inflammatory load aggravating the symptoms. This possible link between periodontal disease giving rise to schizophrenia cannot be overlooked due to the presence of cytokine activity which is present both in schizophrenia and periodontal disease [20]. A study by Dana Graves, has explained the role of chemokines on periodontal health in schizophrenic patients which may directly or indirectly modulate leukocyte recruitment and osteoclast formation [29]. Greater probing depths and clinical attachment loss in schizophrenic patients found in this study suggesting greater periodontal destruction could be the result of exposure to cytokines such as interleukins and other inflammatory mediators produced in the pathophysiologic course of schizophrenia. Alterations in inflammatory related genes, genetic polymorphism of cytokines, proinflammatory cytokine dysregulation including upregulation in levels of IL-1 $\beta$, IL-6, IL-9, TNF- $\alpha$, TNF- $\beta$, PGE2 and CRP in schizophrenic patients can adversely influence the periodontal disease and its progression [30,31]. The continued presence of inflammatory cytokines due to periodontal disease may have an influence on neurotransmitter mechanism in schizophrenia by modulating the dopaminergic metabolism thereby negating the effects of medication. IL-1 $\beta$, a key cytokine in periodontal disease, has been shown to affect the neurotransmitters by enhancing dopamine survival and inhibiting glutamate release leading to hypofunction of N-Methyl-D-Aspartate (NMDA) glutamate receptors which may lead to schizophrenia. Similarly, elevated IL-6 levels, another cytokine implicated in periodontal disease, have been shown to be associated with duration of illness of schizophrenia [32-34]. This cytokine dysregulation may stem from psychotic drugs and/or immunological phenotype of schizophrenia.

As evidenced by the literature, systemic conditions pose greater risk for periodontal attachment loss as well share bilateral relationships. The findings of this study strengthen the relationship between systemic and periodontal diseases and can draw attention of dentists, periodontists and psychiatrists towards the association between schizophrenia and periodontal disease. And also forms basis to set the clinical guidelines for the management of periodontal diseases in schizophrenic patients. Understanding this association can enable the dentists and periodontists to structure and implement effective periodontal treatment plan.

The schizophrenic condition can possibly influence the prognosis of dental treatments, such as periodontal treatment, endodontic therapy, extraction etc. A case report by Lizett Castellanos-Cosano et al., showed no post operative complications following dental implant placements and it can be a suitable option for schizophrenic patients under psychiatrist's consultation [35]. However, there is scarcity of literature that assess the influence of schizophrenia on various dental treatments and there is necessity of more researches to explore this aspect. A systematic review suggests that patients with schizophrenia have worse oral health than the general population, but have received less dental care services and the oral health services should be taken into account in the patients with schizophrenia [36]. Providing oral care for patients with schizophrenia can be challenging, because this disorder can prevent the patient from mindful participation in oral care which can be further compounded by the presence of motor, cognitive, social and behavioural impairments [37]. The dentists should consider the possible influence of patients' cognitive status, effects of antipsychotic drugs and possible interactions with dental drugs. Dentists must be familiar with the disease process so that they can communicate effectively with the patient, the treating psychiatrist and family members who serve as caregivers. [38].

The treatment interventions in schizophrenia may have influence on the association of this complex psychosis with periodontal disease. Hence, absence of drug free schizophrenic control group forms the limitation of this study. However, this study is the first of its kind that explored the relationship between schizophrenia and periodontal disease in relation to cortisol levels eliminating the confounders, such as stress, xerostomia and tobacco consumptions. Further longitudinal clinical studies are warranted to strengthen the causal-effect relationship between schizophrenia and periodontal disease.

\section{Conclusions}

Our study establishes the association between schizophrenia and periodontal disease which is bilateral. There is no significant role of cortisol and other shared risk factors in schizophrenic patients undergoing treatment indicating the possible immunoinflammatory dysregulation. Findings of this study, provides ground evidence for consideration of periodontitis and schizophrenia as mutual risk factor and demands greater emphasis on periodontal care of schizophrenic patients similar to other comorbid disorders and also incorporating periodontal care measures in the clinical guidelines for schizophrenia management.

\section{Abbreviations}

ELISA: Enzyme linked immunosorbent assay; GI: Gingival index; PI: Plaque index; PD: Probing depth; CAL: Clinical attachment loss; L: Interleukin; TNF: Tumour necrosis factor; PGE: Prostaglandins E; CRP: C reactive protein. 


\section{Acknowledgements \\ None.}

\section{Authors' contributions}

All the authors have contributed for conception of research, data procurement, data analysis, manuscript writing and approval. All authors read and approved the final manuscript.

\section{Funding}

No funding was received for this research.

\section{Availability of data and materials}

Study related data and materials are available and can be provided on request, if needed.

\section{Declarations}

\section{Ethics approval and consent to participate}

Study protocol is in accordance with revised 2013 Helsinki declaration for experimentations involving human subjects and approved by ethical research committees of College of Dentistry, Qassim University and Al Amal \& Psychiatric Hospital, Buraidah with number F-3003-18. Informed written consent is obtained from the study participants and their caretakers.

\section{Consent for publication}

All the authors have consented for the publication.

\section{Competing interests}

There are no competing interest related to this study.

\begin{abstract}
Author details
'Pediatric Dentistry Resident, Ministry of Health, Al Qassim, Saudi Arabia. ${ }^{2}$ Dentist Resident, Ministry of Health, Riyadh, Saudi Arabia. ${ }^{3}$ Department of Periodontology and Oral Medicine, College of Dentistry, Qassim University, Buraidah, Al Qassim, Kingdom of Saudi Arabia. ${ }^{4}$ Department of Dental Hygiene, College of Applied Health Sciences in Ar Rass, Qassim University, 51921 Al Qassim, Kingdom of Saudi Arabia.
\end{abstract}

Received: 26 August 2021 Accepted: 25 November 2021

Published online: 11 December 2021

\section{References}

1. Friedlander A, Marder S. The psychopathology, medical management and dental implications of schizophrenia. J Am Dent Assoc. 2002;133:603-10.

2. Sadock BJ, Sadock VA. Kaplan and Sadock's synopsis of psychiatry. Williams and Wilkins, New York. 2007. 10th Edition.

3. World Health Organization. Schizophrenia. WHO. 2018: https://www.who. int/news-room/fact-sheets/detail/schizophreni

4. Wey M, Loh S, Doss J, Abu Bakar A, Kisely S. The oral health of people with chronic schizophrenia: a neglected public health burden. Aust N Z J Psychiatry. 2015:50:685-94.

5. American Psychiatric Association. Diagnostic and statistical manual of mental disorder. 2000 4th edition. Washington DC.

6. Shreya S, Bose A. Schizophrenia and periodontal disease: An oro- neural connection? A cross-sectional epidemiological study. J Indian Soc Periodontol. 2014;18:69-73.

7. Đorđević V, Dejanović S, Janković L, Todorović L. Schizophrenia and oral health—review of the literature. Balkan J Dent Med. 2016;20:15-21.

8. Leucht S, Burkard T, Henderson J, Maj M, Sartorius N. Physical illness and schizophrenia: a review of the literature. Acta Psychiatr Scand. 2007;116:317-33.

9. Jayanthi M, Faizuddin M, Noor-Ahamadi HM. Association of interferon lambda-1 with herpes simplex viruses-1 and -2, Epstein-Barr virus, and human cytomegalovirus in chronic periodontitis. J Investig Clin Dent. 2017. https://doi.org/10.1111/jicd.12200.

10. Kinane DF. Causation and pathogenesis of periodontal disease. Periodontol. 2000;2001(25):8-20
11 Rodríguez Franco NI, Rubia J. Plaque index, oral hygiene habits, and depressive symptomatology as predictors of clinical attachment loss: a pilot study. Int J Dent. 2020. https://doi.org/10.1155/2020/3257937.

12. Löe $H$. The gingival index, the plaque index and the retention index systems. J Periodontol. 1967:38:610-6.

13 Tonetti MS, Greenwell H, Kornman KS. Staging and grading of periodontitis: framework and proposal of a new classification and case definition. J Periodontol. 2018;89(1):S159-72.

14 Papapanou PN, Sanz M, Buduneli N, Dietrich T, et al. Periodontitis: consensus report of workgroup 2 of the 2017 world workshop on the classification of periodontal and peri-implant diseases and conditions. J Clin Periodontol. 2018:45(S20):S162-70.

15. Arnaiz A, Zumárraga M, Díez-Altuna I, Uriarte J, Moro J, Pérez-Ansorena M. Oral health and the symptoms of schizophrenia. Psychiatry Res. 2011;188:24-8.

16. Hugoson A, Ljungquist $B$, Breivik T. The relationship of some negative events and psychological factors to periodontal disease in an adult Swed ish population 50 to 80 years of age. J Clin Periodontol. 2002;29:247-53.

17 Bozovic D, Racic M, Ivkovic A. Salivary cortisol levels as a biological marker of stress reaction. Med Arch. 2013;67:374-7.

18. Cabral DM, Antonini SR, Custódio RJ, Martinelli CE Jr, da Silva CA. Measurement of salivary cortisol as a marker of stress in new born in a neonatal intensive care unit. Horm Res Paediatr. 2013;79:373-8.

19. Zoja B, Branko S, et al. Serum cortisol in patients with schizophrenia: association with psychopathology and response to antipsychotics. Sylwan J. 2015;59:421-34.

20. Kornetova E, Semke A, Kornetov A, Dmitrieva E, Dubrovskaya V, Bokhan N. Morphophenotypical patterns in patients with negative symptoms in schizophrenia. Euro Psych. 2017:41:S818.

21 Gupta S, Gupta R. Necessity of oral health intervention in schizophrenic patients—a review. Nepal J Epidemiol. 2017;6:605-12.

22. Mann K, Rossbach W, Muller M, Mullersiecheneder F, Pott T, Linde I, et al, Nocturnal hormone profiles in patients with schizophrenia treated with olanzapine. Psychoneuroendocrinol. 2006;31:256-64.

23. Ryan M, Sharifi N, Condren R, Thakore J. Evidence of basal pituitary-adrenal overactivity in first episode, drug naïve patients with schizophrenia. Psychoneuroendocrinology. 2004;29:1065-70.

24. Rady A, Elsheshai A, Elsheikh M, Eltawel M. Long term use of antipsychotics in schizophrenia and relation to cortisol level. Euro Psych. 2015;30:1730.

25 Veerabhadrappa S. Evaluation of xerostomia in different psychologica disorders: an observational study. J Clin Diagn Res. 2016:10:24-7.

26. Longman L, McCracken C, Higham S, Field E. Clinical Oral Medicine: the clinical assessment of oral dryness is a significant predictor of salivary gland hypofunction. Oral Dis. 2008;6:366-70.

27. Gelder M, Harrison P, Cowen P. Symptoms and signs of psychiatric disorders Textbook of psychiatry 5th edition. New York: Oxford, 2008, pp 1-20.

28. Eltas A, Kartalcı Ş, Eltas Ş, Dündar S, Uslu M. An assessment of periodontal health in patients with schizophrenia and taking antipsychotic medication. Int J Dent Hyg. 2013;11:78-83.

29. Graves D. Cytokines that promote periodontal tissue destruction. J Periodontol. 2008;79:1585-91

30. Saetre $P$, Emilsson $L$, Axelsson $E$, Kreuger J, Lindholm E, Jazin E. Inflammation-related genes up-regulated in schizophrenia brains. BMC Psychiatry. 2007:7:46.

31. Meyer U, Schwarz MJ, Müller N. Inflammatory processes in schizophrenia: a promising neuroimmunological target for the treatment of negative/ cognitive symptoms and beyond. Pharmacol Ther. 2011;132:96-110.

32. Song C, Li X, Kang Z, Kadotomi Y. Erratum: Omega-3 fatty acid ethyleicosapentaenoate attenuates il-1 $\beta$-induced changes in dopamine and metabolites in the shell of the nucleus accumbens: involved with PLA2 Activity and Corticosterone Secretion. Neuropsychopharmacol. 2007;32:1207.

33. Gani D, Lakshmi D, Krishnan R, Emmadi P. Evaluation of C-reactive protein and interleukin-6 in the peripheral blood of patients with chronic periodontitis. J Indian Soc Periodontol. 2009;13:69-74.

34. Ganguli R, Yang Z, Shurin G, Chengappa K, Brar J, Gubbi A, et al. Serum interleukin-6 concentration in schizophrenia: elevation associated with duration of illness. Psych Res. 1994;51:1-10. 
35 Castellanos-Cosano L, Corcuera-Flores J, et al. Dental implants placement in paranoid squizofrenic patient with obsessive-compulsive disorder: a case report. J Clin Exp Dent. 2017:9:e1371-4.

36 Yang M, Chen P, He M-X, et al. Poor oral health in patients with schizophrenia: a systematic review and meta-analysis. Schiz Res. 2018;201:3-9.

37. da Purkayastha SM, Gurenlian JR. Oral health care for patients with schizophrenia. J Multi Care. 2020;6:40-4.

38 Friedlander AH, Marder SR. The psychopathology, medical management and dental implications of schizophrenia. J Am Dent Assoc. 2002;133:603-10

\section{Publisher's Note}

Springer Nature remains neutral with regard to jurisdictional claims in published maps and institutional affiliations.

\section{Submit your manuscript to a SpringerOpen ${ }^{\circ}$ journal and benefit from:}

- Convenient online submission

- Rigorous peer review

- Open access: articles freely available online

- High visibility within the field

- Retaining the copyright to your article

Submit your next manuscript at $\boldsymbol{\nabla}$ springeropen.com 